Materials Horizons

\title{
Two-dimensional Arrays Self-assembled via Interference of Concentration Modulation Waves in Drying Solution $\dagger$
}

Received 00th January 20xx, Accepted 00th January 20xx

DOI: $10.1039 / x 0 x \times 00000 x$

www.rsc.org/
Shunpu Li, $\ddagger^{\mathrm{ab}}$ Young Tea Chun, $\ddagger^{\mathrm{a}}$ Jin Li, ${ }^{\mathrm{a}}$ Pawan Shrestha ${ }^{\mathrm{a}}$, Hyungju Ahn, ${ }^{\mathrm{c}}$ Docheon Ahn, ${ }^{\mathrm{c}}$ Jung Inn Sohn, ${ }^{d}$ Woong-Ki Hong, ${ }^{e}$ Bongjun Kim, ${ }^{f}$ Yuanbo Deng ${ }^{a}$ and Daping Chu*a
Self-assembling of patterned nanostructures in solution-processed thin films with multiphase block-copolymers is possible. Generation of small and well-defined regular structures directly from single-phase polymer films is challenging. Here, 2-dimensional (2D) patterned array in single-phase polymers was self-assembled by a solution process. One-dimensional spinodal precipitation with a characteristic wavelength was created along a pinned contact line of the drying solution to form a one-dimensional periodic structure (1DPS) via geometry and concentration confinements. The latter was uncovered from the liquid by sequential depinning and repinning of the contact line. A new 1DPS with an inherited phase re-emerged and triggered depinning and repinning. The process was self-repeated and a $2 \mathrm{D}$ array formed with a tuneable lattice type and parameters, which was governed by the interference of two concentration-modulated waves.

\section{Introduction}

Self-assembled nanometer-length patterns in two dimensions (2D) are of considerable interest for several applications, such as lithography with high resolution and optical devices. Selfassembly in 2D has been studied in self-assembled monolayers, quantum dots and colloidal particles. ${ }^{2,3}$ Among these systems, the self-assembly of thin-structured polymer films has many attractive aspects because it is inexpensive and compatible with conventional lithography processes such as thin-film coating

\footnotetext{
a. Department of Engineering; University of Cambridge, $9 \mathrm{JJ}$ Thomson Avenue, Cambridge, CB3 OFA, UK. *Corresponding author e-mail: dpc31@cam.ac.uk

b. College of New Materials and Energies, Shenzhen Technology University, Shenzhen, 518118, China.

Beamline Department, Pohang Accelerator Laboratory; POSTECH, Gyeongbuk, Pohang, 37673, South Korea.

d. Division of Physics and Semiconductor Science, Dongguk University, Seoul, 04620, South Korea.

e. Jeonju Center, Korea Basic Science Institute Jeonju-si, Jeollabuk-do, 54896, South Korea.

f. Department of Electronics Engineering, Sookmyung Women's University, Seoul, 04310, South Korea.

† Electronic Supplementary Information (ESI) available. See DOI: 10.1039/x0xx000

₹ These authors contributed equally
}

\section{Conceptual insights}

A long-standing challenge in nanofabrication with self-assembling technique is to assemble of two-dimensional array with single-phase polymer. Attempt has been made to generate such 2D-array from solution, like monodispersed water droplet condensation onto evaporating solution; generation of line pattern from drying solution and subsequent dewetting the lines into dot array. However, these existing approaches are difficult for patterning $2 \mathrm{D}$-array with high resolution. Here, we demonstrate a concept to fabricate $2 \mathrm{D}$-array by using interference between two concentration-modulation-waves. The principle is somehow in analogy to optical interference lithography, but the two plane waves are formed via solute concentration-modulation in drying solution next to the contact line: One wave is originated from solvent evaporation induced solute-condensation at drying front with wave vector perpendicular to the contact line; while, another wave involved is spinodal-precipitation induced by constitutional undercooling with wave vector along the contact line. The lattice parameter of the array can be controlled down to deep sub-micrometre and the lattice type could be tuned, like square and hexagonal. With further development, this work may open a new way to produce low-cost 2D self-assembled crystals for certain applications, such as opto-electric devices, lithography template, photonic materials etc.

and pattern transfer with dry etching. The self-assembly of block-copolymer thin films has received considerable attention due to the intrinsic ability of the materials to generate arrays of one phase distributed in another matrix phase. ${ }^{4-6}$ After selective removal of one block-phase, the remaining patterned phase can be transferred into any functional material. 7,8 In contrast, the generation of long-range periodic structures with high resolution from single-phase polymer thin films is challenging. Attempts have been made to generate $2 \mathrm{D}$ arrays of single-phase polymers through liquid processes. Condensation of monodispersed water droplets onto evaporating solutions has be used to generate "honeycomb structures" that have sizes limited to micrometers. ${ }^{9-11}$ Generation of line patterns from a drying solution and subsequent dewetting the lines into dots have also been demonstrated. ${ }^{12,}{ }^{13}$ However, the dewetting approach is unlikely to be adopted to fabricate structures with high resolution (deep sub-micrometer or nanometer) over a large area because material tearing induces many defects and nonuniformity. 
Here, we demonstrate a pattern-formation process that allows generation of 2D arrays with deep sub-micrometre. The principle can be analogized to optical interference lithography, ${ }^{14,15}$ but the two waves are formed via modulation of concentration in the drying solution next to the three-phase line (or contact line). One wave originates from solvent evaporation-induced solute-condensation (SC) next to the contact line with wave vectors perpendicular to the contact line. Another wave involved here is spinodal-precipitation induced by constitutional undercooling with its wave vector along the contact line.

\section{Results and discussion}

We considered two phenomena often encountered in thin-film processes with solutions to elicit the two waves that participate in pattern formation. A solution is confined to a particular geometry, for instance, in a wedge-shaped space formed by a substrate and a cover plate (Figure. 1A). When the contact line between the substrate and solution is pinned, the liquid that evaporates from the contact line is replenished by liquid from the interior, so that an outward flow carries the solute to the contact line (coffee-stain effect). ${ }^{16}$ The contact angle of the solution with the substrate, $\vartheta$, is not unique but can vary over a finite range. The surface topography of substrate influences the range of the contact angle. ${ }^{17}$ When the solvent evaporates, the contact line will not recede on a flat substrate surface until the contact angle decreases to a critical receding contact angle $\vartheta_{r}$ (i.e., $\vartheta \leq \vartheta_{r}$ ). ${ }^{18}$ Solute deposition next to the contact line by the coffee-stain effect (its cross-section is illustrated as a small "dome" in Figure 1A) causes a reduction of the local contact angle $\vartheta^{*}$ (red arrow in Figure 1a) to a value below the $\vartheta_{r}$ $\left(\vartheta^{*} \leq \vartheta_{\mathrm{r}}\right)$, and depinning of the contact line begins. The depinned contact line slides over the deposited solute line and is pinned again at the shoulder of the solute line, where the maximum slope is found, ${ }^{19}$ until the next solute line is deposited and developed. ${ }^{20,21}$ The line patterns defined by the pinningdepinning mechanism can be found through drying polymers or colloids from solutions. 13, 20, 22 Another phenomenon we consider here is spinodal-precipitation. Figure $1 \mathrm{~B}$ is a schematic illustration of the phase diagram of a binary mixture (such as a solvent-polymer system). The binodal and spinodal curves are defined by $\partial G / \partial C=0$ and $\partial^{2} G / \partial C^{2}=0$, where $G$ and $C$ are Gibbs' free energy and polymer concentration, respectively. For concentrations within the spinodal region, where $\partial^{2} G / \partial C^{2}<0$, a homogeneous solution is unstable against infinitesimal fluctuations in density or component concentration, and separates to two phases with a well-defined size (i.e., spinodal wavelength $\lambda_{s n}$ ) with no thermodynamic barrier (inset of Figure 1B). ${ }^{23}$

The interference between the solute condensation (SC) wave induced by the coffee-stain effect and the spinodal (SN) wave occurs next to the contact line is understood less intuitively in comparison with what happens with optical and liquid-surface waves. However, the pattern-formation process can be appreciated through description of the generation and reproduction of a 1D periodic structure (1DPS). The latter is realized through 1D-spinodal (1DSN) precipitation along the contact line via restriction of geometry and concentration (inset of Figure 1A). By coupling such a 1DPS formation with the repeated stepwise pinning-depinning of the contact line, a 2D pattern can be generated (Figure 1C). The 1DPS is formed next to the contact line in the solution (red circles in Figure $1 \mathrm{C}$ at the moment $t_{0}$ ), which reduces the local contact angle of the solution and triggers depinning of the contact line to move forward for a step-length $\lambda_{c l}$, where it is repinned. Meanwhile, a new 1DPS emerges and develops ( $t_{1}$ of Figure $1 C$ ), and triggers the depinning of the contact line again ( $t_{2}$ of Figure $\left.1 C\right)$. The process is self-repeating and a $2 \mathrm{D}$ array is formed.

We attempted to fabricate such 2D arrays from solutions through tuning of the solution concentration, solutionconfinement geometry, and drying condition. There are three main reasons to support this idea. First, with solvent evaporation, the solution in the vicinity of the contact line is concentrated and can be located in the spinodal region $\left(C_{1}\right.$ in Figure $1 B$ ), whereas the bulk liquid remains stable ( $C_{0}$ is outside the spinodal region, Figure $1 \mathrm{~B}$ ). Such a solute condensation (from $C_{0}$ to $C_{1}$ ) near the contact line defines a driving force (i.e., $\Delta T$, which is well known as "constitutional undercooling" in metallurgy ${ }^{24}$ ) for spinodal precipitation. Second, for a polymersolvent binary system, the binodal and spinodal curves are very close to each other at the solvent side, and this minimizes the impact of the binodal region where phase separation occurs via nucleation. ${ }^{25}, 26$ Third, the spinodal-wave generation in a solution is extremely sensitive to concentration fluctuations, so a new 1DPS (red circles in Figure $1 \mathrm{C}$ ) are initiated from the nearby solution with concentration modulation caused by a previously formed 1DPS (neighbouring magenta circles). Thus, two neighbouring 1DPSs should be phase correlated ( $\Delta \phi=$ constant), which is crucial for assembling an ordered $2 \mathrm{D}$ structure. For instance, if $\Delta \phi=0$, a rectangular (or square) array will be formed, and if $\Delta \phi=\pi$, a hexagonal array is expected. For sample preparation (Supporting Information Note 1), $60 \mu \mathrm{L}$ of polymer solution was introduced into a wedge-shaped space, formed with a silicon substrate $(12 \mathrm{~mm} \times 12 \mathrm{~mm})$ and a glass cover plate $(12 \mathrm{~mm} \times 12 \mathrm{~mm})$ with an angle of $25^{\circ}$, to create a stable liquid bridge. The substrates were treated by $\mathrm{O}_{2}$ plasma (100 $\mathrm{W}$ for $5 \mathrm{~min}$ ) to increase the binding strength of the formed polymer patterns on them. Polymer concentrations of the bulk solutions were optimized at $0.5-5 \mathrm{mg} / \mathrm{ml}$. Drying was carried out in a fume hood to allow the solvent to evaporate steadily with an estimated mean moving speed of the solution meniscus of $\approx 4.5 \mu \mathrm{m} / \mathrm{s}$. Figure 2 displays images of the self-assembled patterns in films dried from toluene solutions of poly(4emethylstyrene) (PMS, $5 \mathrm{mg} / \mathrm{ml}$ ), polystyrene (PS, $3.7 \mathrm{mg} / \mathrm{mL}$ ), and Poly\{[N, $N^{\prime}$-bis(2-octyldodecyl)-naphthalene-1,4,5,8bis(dicarboximide)-2,6-diyl]-alt-5,5'-(2,2'-bithiophene)\}

[P(NDI2OD-T2), $2.5 \mathrm{mg} / \mathrm{ml}$ ]. Obviously, regular patterns were generated and all patterns had near four-fold symmetry. The typical thickness of the deposited polymer films was 30-100 $\mathrm{nm}$, and the amplitudes of the generated patterns were in the same scale as measured with atomic force microscopy (AFM) (Figure $3 \mathrm{~A}$ ). The AFM result showed that each peak was neighboured by four valleys and four saddle-points, which is a 
A

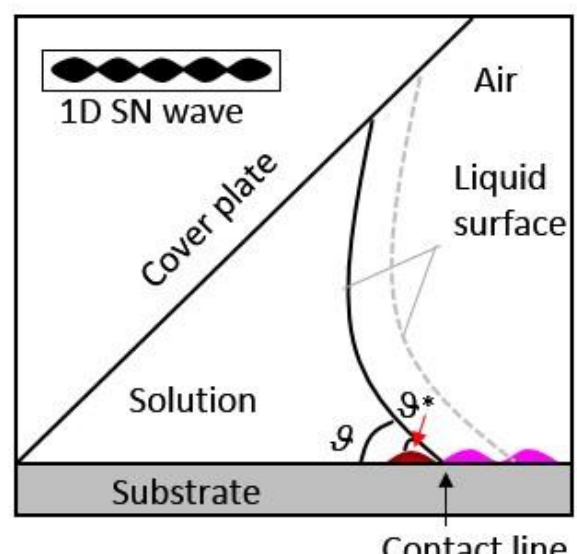

C

Contact line

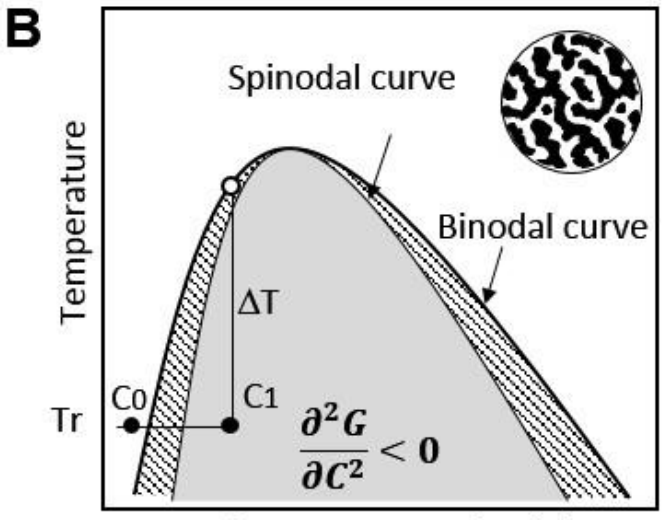

Solute concentration (C)
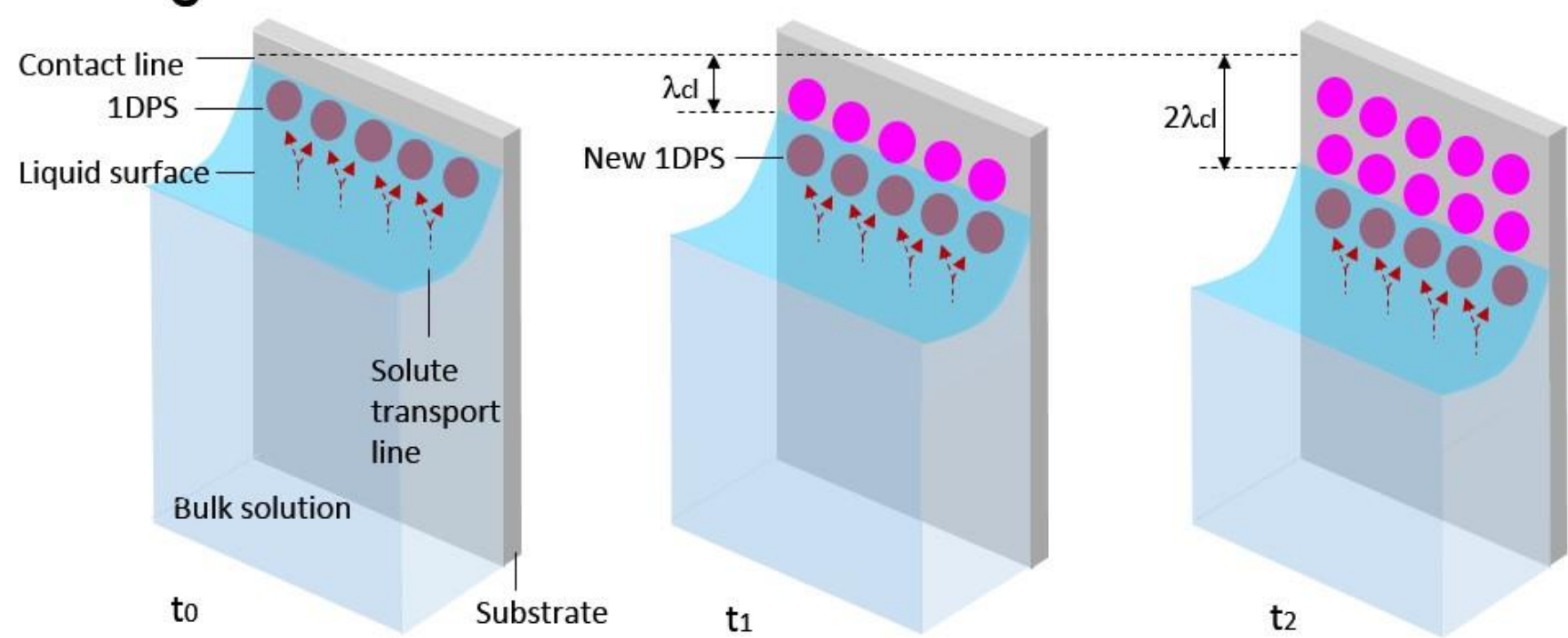

Fig. 1 Principle of 2D-array generation from solution (schematic). (a) Pinning-depinning of a contact line between the substrate and solution constrained in a wedge-shaped space (schematic). A red dome represents the cross-section of the precipitated solute line during deposition. Magenta domes represent the solute lines that have been generated. The inset is a schematic drawing of 1D spinodal (1DSN) concentration fluctuation confined along the contact line when a certain condition is satisfied. (b) Schematic diagram of spinodalprecipitation in a binary system, where Tr represents room temperature. The inset is a schematic drawing of microstructure formed through spinodal precipitation. (c) Schematic illustration of formation of a 2D array. Red circles represent a 1DPS under development in solution, whereas the magenta circles show that 1DPSs have been generated.

strong signature of the interference of the two orthogonal plane waves (explained in Figure S1). If we consider the interference between two orthogonal plane waves with wave vectors $k_{x}=2 \pi / \lambda_{s n}$ and $k_{y}=2 \pi / \lambda_{c l}$, the surface profile of the $2 D$ patterns can be modeled by:

$$
\mathrm{z}=\frac{\xi}{2}\left(\cos \frac{2 \pi x}{\lambda_{s n}}+\cos \frac{2 \pi y}{\beta \lambda_{s n}}\right)
$$

where $\xi, \lambda_{s n}$ and $\lambda_{c l}$ are the peak-valley distance, lattice parameter along the contact line (i.e. spinodal-wavelength), and step length of contact line movement (i.e. SC-wavelength or depinning wavelength), respectively, whereas $\beta=\lambda_{c l} / \lambda_{s n}$, which is close to one. $x$ and $y$ are taken along the direction of the contact line and its orthogonal direction in the substrate surface plane.

The agreement between the modelling and AFM measurement (Figure $3 \mathrm{~A}$, and a quantitative comparison as detailed in Figure S2) also supports the existence of wave interference. The lattice parameters of the patterns varied for different polymers, which are explained by the difference in polymer-chain mobility in the solutions. The spinodalwavelength can be expressed by: ${ }^{23}$

$$
\lambda_{s n}=\sqrt{8 \pi^{2} k M / D}
$$

where $D, M$ and $k$ are the diffusion coef $f_{f}$ icient, solute mobility and a constant, respectively. According to the Stokes-Einstein relationship: ${ }^{27} \mathrm{D}=\mathrm{k}_{\mathrm{B}} \mathrm{T} / 6 \pi \eta \mathrm{R}$ (where $\mathrm{T}, \eta, R$, and $k_{B}$ are absolute temperature, solution viscosity, polymer radius of gyration and the Boltzmann constant, respectively) and the Walden-Adamczewski formula:28-30 $M \eta^{m}=$ constant (where $m$ is a constant), we obtain:

$$
\lambda_{s n}=\sqrt{48 \pi^{3} \delta R / k_{B} T \eta^{m-1}}
$$




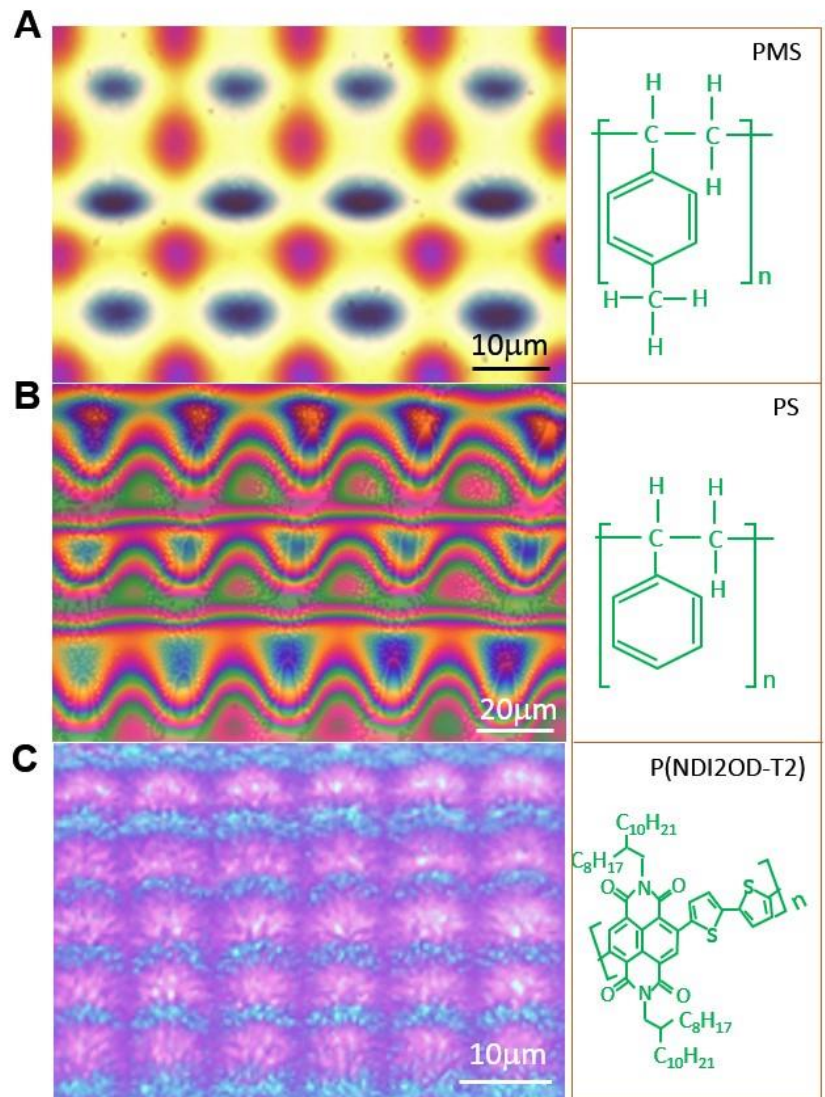

Fig. 2 Optical images of self-assembled 2D patterns in films of various polymers. (a) PMS (b) Polystyrene; (c) P(NDI2OD-T2). For all images, the contact lines were aligned in the horizontal direction and moved in the vertical direction (from top to bottom).

where $\delta$ is a constant, and it is clear that the solution viscosity influences the spinodal wavelength. Figure $3 \mathrm{~B}$ displays the measured lattice parameters of arrays and solution viscosity of different systems (top panel), demonstrating that a highviscosity solution is favourable for obtaining a pattern with a smaller period. By fitting the experimental data with a logarithmic plot (Figure 3B, bottom panel), we obtain $m \approx 2.5$, and this supports the hypothesis that the pattern formation originates from spinodal-precipitation. Equation 3 also supports the small influence of temperature on the spinodal-wavelength we observed (inset of Figure 3B), which is explained in Supporting Information (Note 2).

The obtained near-square lattices suggest that the spinodalwavelength $\left(\lambda_{s n}\right)$ is close to the step-length of the contact line movement $\left(\lambda_{\mathrm{cl}}\right)$, i.e., $\lambda_{\mathrm{sn}} \approx \lambda_{\mathrm{cl}} \approx \lambda$. To explain this phenomenon, one can speculate that the front 1DPS is formed from polymer clusters chaining-up along the contact line, a hypothesis that is supported by a morphology study on spinodal-decomposition in a highly confined solution. ${ }^{[31]}$ To verify this hypothesis, we quenched a sample by suddenly flushing away the solution with 2-propanol during 2D PMS pattern growth from its toluene solution (Figure 3C). It is clear that a chain of "domes" along the contact line forms the front 1DPS in the solution (indicated by a red arrow). The modulated dark shadow ahead of the front 1DPS (indicated by a black arrow) signifies that the concentration-modulation rooted from the front 1DPS projects into a certain depth in the solution, which is the scale of the undercooled liquid caused by polymer condensation. The growth of domes is much faster than the precipitation that occurred in the deeper region of the liquid because the solute concentration defined by solid-liquid interface diffusion and hydrodynamic condensation are much higher at the contact line. The dome-shape is resulted from minimization of the surface energy of the precipitated "half-solid" elastic polymer. The co-existence of the two decoupled waves and their interference can occasionally be observed on "unsuccessful" samples where local fluctuation induces an imbalance of the amplitudes of the two waves (Figure S3). The process of 1DPS formation has also been further demonstrated with in-situ examination of solution drying under microscopy (Supporting movie and its annotation in Figure S4). The detailed reason of spinodal and depinning wavelengths similar to each other is still an open question. One possible explanation is that the final spinodal wavelength is resulted from coarsening of initially selected spinodal wave until it matches with the depinning wavelength. This could also explain the coexistence of different spinodal wavelengths on the same sample observed in certain systems (Figure S3). From the thermodynamics point of view, both wavelengths are resulted from the minimization of Gibbs free energy in an identical condition, though the reasons and processes of wave creations are different, and having similar lattice parameters defined by the two waves are plausible.

Strong confinement of a solution favours a reduction of structure dimension of spinodal-precipitated polymers. ${ }^{31}$ This may also apply in our case to form arrays with much smaller lattices. We have introduced P(NDI2OD-T2) solution (in toluene or 1,2-dichlorobenzene, $2.5 \mathrm{mg} / \mathrm{ml}$ ) into a space between a $\mathrm{Si}$ substrate and a cover plate [glass or polydimethylsiloxane (PDMS)] that were aligned parallel and separated using spacers of various thicknesses to examine the effect of solution confinement (inset drawing of Figure 3D). A reduction of spinodal-wavelength was observed when the liquid film thickness was less than $\approx 30 \mu \mathrm{m}$ (black circles in Figure 3D). Square array with deep sub-micrometre lattice parameter was found when the liquid thickness was reduced to $1.5 \mu \mathrm{m}$ (Supporting Information Note 3, inset of Figure 3D). We attempt to explain this size reduction as a pressure change $(\Delta P)$ within the solution caused by an increased Laplace pressure of the liquid/air interface. When the confinement increases, the pressure in the liquid becomes more negative than atmospheric pressure and the density of vacancy (or air holes) in the solution increases, which is favourable for nucleation of the precipitated polymer phase. Based on this assumption, we derived a relationship between the confined liquid-film thickness $h$ and spinodal-wavelength $\lambda_{\text {sn }}$.

$$
\lambda_{s n}=\lambda_{s n}^{b}-\frac{2 \chi \gamma \cos \vartheta}{h+\varepsilon}
$$

where $\lambda_{s n}^{b}, \chi$ and $\varepsilon$ are the spinodal-wavelength for bulk solution and constants, whereas $\gamma$ and $\vartheta$ are the surface tension of the solution and contact angle, respectively. By choosing $\chi=$ $2.77 \times 10^{-9}\left(\mathrm{~m}^{3} / \mathrm{N}\right)$ and $\varepsilon=1.076 \times 10^{-5}(\mathrm{~m})$, equation (4) could fit 
A

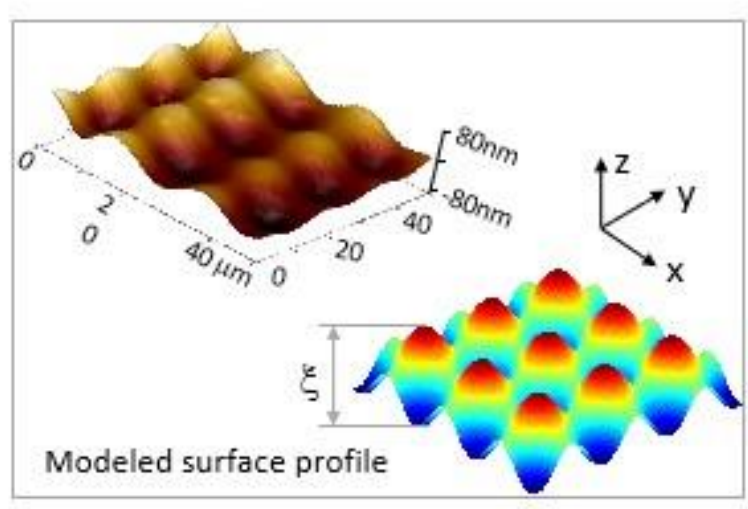

C

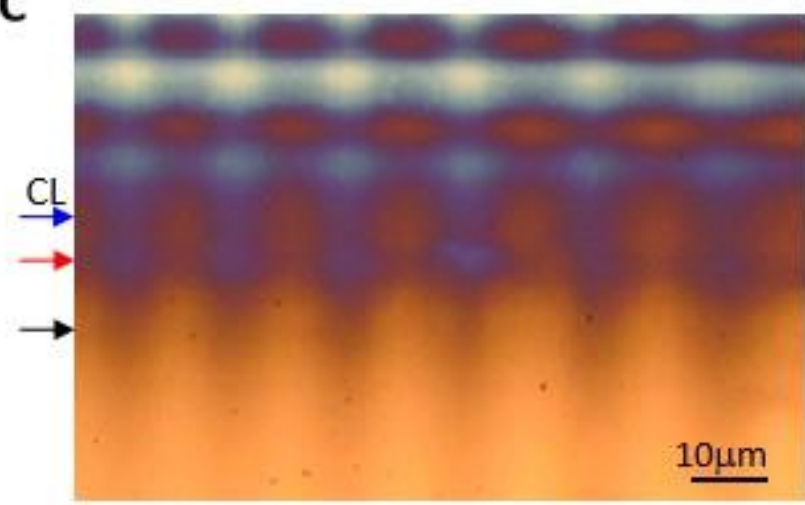

B
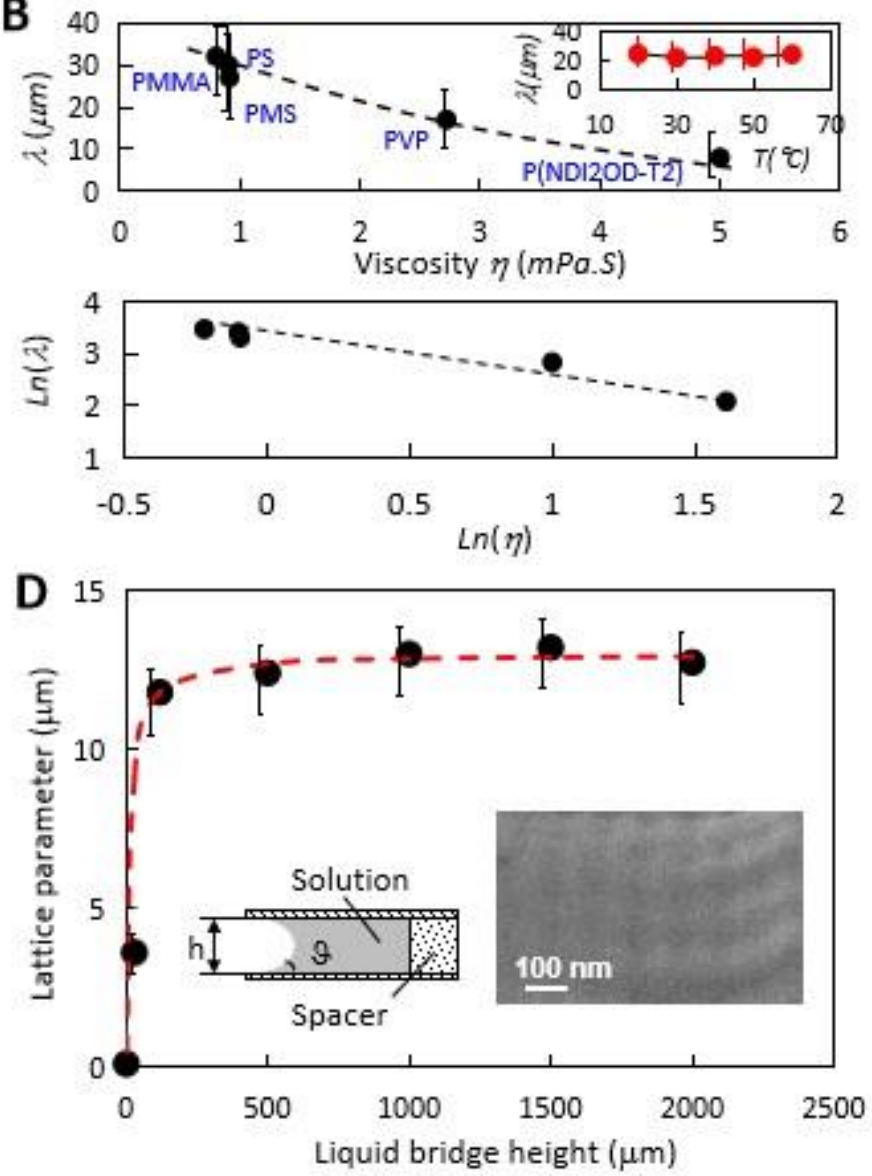

Fig. 3 Pattern analysis and formation mechanism. (a) AFM image taken from the PMS pattern and surface profile modeled with equation (1). (b) Variation in lattice parameters with solution viscosity in different systems, and the temperature dependence of lattice parameters for the PMS pattern from toluene solution (inset). (c) Optical image of a quenched growth front of a 2D array of PMS polymer during growth from its toluene solution. Blue arrow indicates the location of the pinned contact line (CL), and the underdeveloped frontIDPS is indicated by a red arrow. The black arrow indicates a trace of concentration modulation in solution. (d) Solution-thickness dependence of a lattice parameter (or spinodalwavelength) for the P(NDI2OD-T2) structure generated when solutions were confined between parallel plates (inset drawing). SEM image shows a pattern with high resolution (inset SEM).

the experimental data closely (red dotted line in Figure 3d, details in Supporting Information Note 4). The $\varepsilon$ calibrates the deviation of the measured liquid thickness $h$ from the modelled thickness, where the partial cylinder surface is considered as the liquid surface, induced by the surface quality of substrate, mass of liquid, mechanical vibration, etc. The Laplace pressure model as proposed may not be a complete picture as the surface curvature induced pressure difference $(\sim \mathrm{kPa})$ seems to be too small to cause such a drastic wavelength change. Other factors that could reduce the spinodal wavelength, such as the liquid layer thickness next to the contact line, may be important.

Up to now, the $2 \mathrm{D}$ arrays have been shown to be rectangle or near-square. This is because the orientation of the contact line is normally perpendicular to its moving direction, where the solute is moving toward the contact line orthogonally caused by the convection induced by the surface tension gradient. ${ }^{15}$ It is possible to diversify the type of the 2D lattices from rectangular by modifying the angle between the contact line and its moving direction. We have demonstrated this with generation of 2D arrays on glass-tube surfaces by drying a solution confined between the tube and a cylinder container (Supporting Information Note 5). When the glass tube is off-centred in the container, a liquid 'hump' is formed in the gap because of the capillary effect (left of Figure 4A). With evaporation of the solvent, the inclined contact line moves downwards, and a nonorthogonal relationship between the orientation of the contact line and its moving direction is created. The top panel of Figure $4 \mathrm{~B}$ shows a near-hexagonal PMS pattern on the tube surface created with this way. In contrast, for a well-centred tube (middle panel of Figure 4A) the obtained PMS patterns always have near-square symmetry (bottom panel of Figure $4 B$ ).

Once the solvent is dried off the patterned structure is stable. No obvious pattern blurring has been observed after storing the patterned samples in ambient condition for one year. Binding strength of the generated pattern to substrate is still a problem, and it varies from system to system. For example, the PMS pattern dried from toluene solution has much better binding property than other systems we have tested. Currently, we could obtain such 2D arrays over an area of several square millimeters, which is sufficiently large to 
A

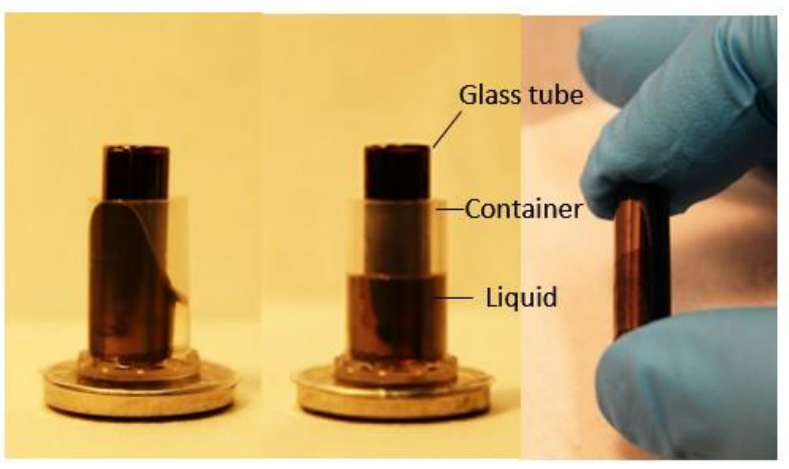

c

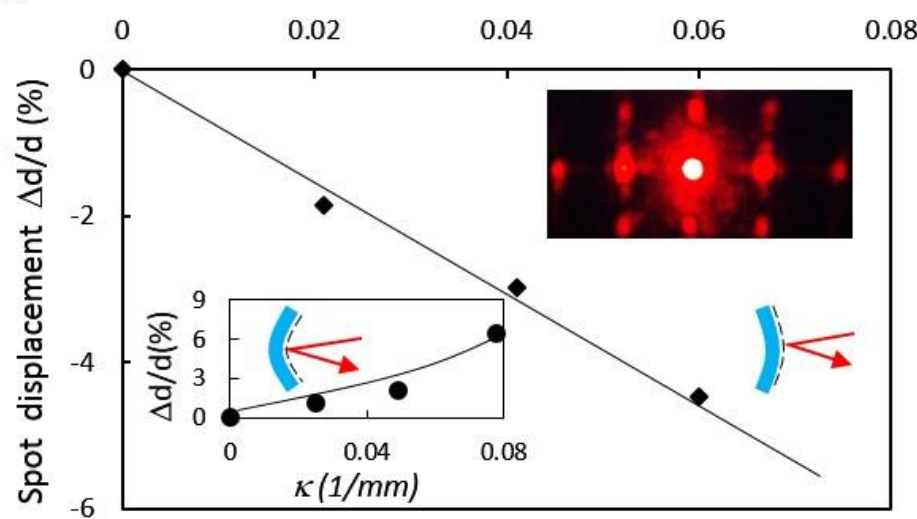

B

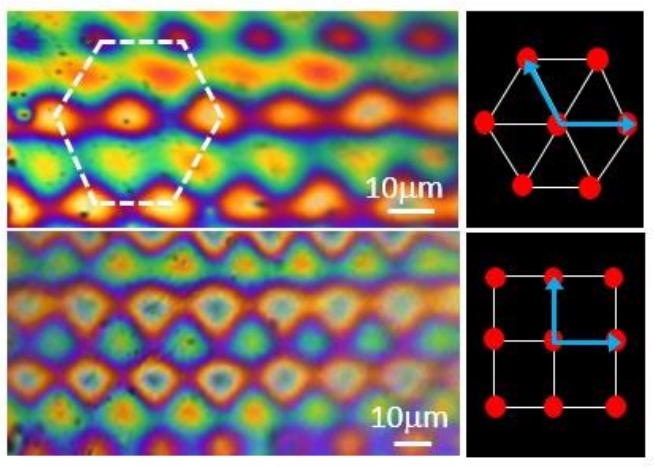

D

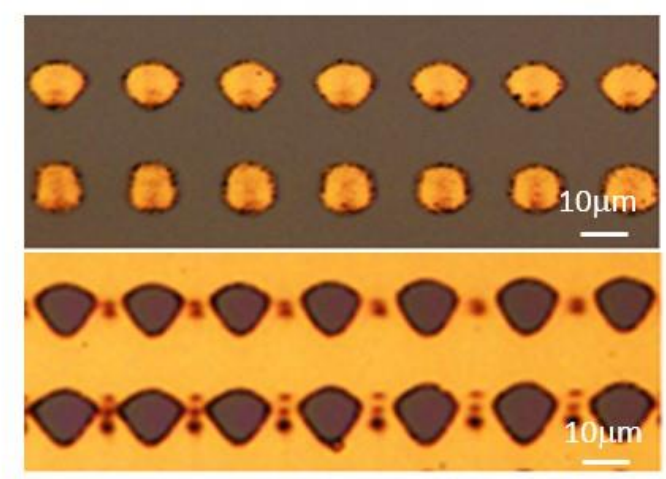

Fig. 4 Lattice-type tuning and potential application of the generated arrays. (a) Optical images of pattern generation on tubes (left panel: off-centred glass tube; middle panel: wellcentred glass tube; right panel: patterned tube); (b) PMS patterns with near-hexagonal (top panel) and near-square (bottom panel) lattices generated on glass-tube surfaces; (c) Diffraction pattern (top inset) and strain-sensing for a patterned surface under tension strain (main panel) and compression strain (bottom inset), where $d$ is the distance between zero and first-order diffraction; (d) Optical images of dot (top panel) and hole (bottom panel) arrays of Au defined by transferring PMS patterns with Au.

demonstrate some functions. Figure $4 \mathrm{C}$ shows a diffraction pattern taken from a PMS array assembled from its toluene solution and demonstration of its strain sensing with the generated pattern acts as a surface-labeled 2D grating on a polyethylene terephthalate (PET) substrate (Supporting Information Note 6, Figure S6). The surface profile of the 2D pattern offers an advantage for pattern transferring: positive and negative arrays can be generated from the same assembled polymer pattern by control of dry etching. If the etching is stopped below the saddle points, a hole-pattern will be defined (Figure S7a and Figure S7b). In contrast, a dot pattern is obtained if the etching is stopped above the saddle points (Figure S7c). Figure 4D shows the dot (top panel) and hole (bottom panel) patterns of Au defined by transferring the selfassembled PMS patterns into polydimethylglutarimide (PMGI) films and subsequent lift-off with Au (Supporting Information Note 7, Figure S8).

Patterns with deep sub-micrometre resolution were generated in a micro-sized environment where a significant suppression of solution convection is expected. However, a hydrodynamic process is necessary to achieve sufficient concentration confinement next to the contact line and in turn to realize spinodal-precipitation at sub-micrometre scale. To gain some information about the hydrodynamic influence during solution drying under strong confinement, we undertook grazing-incidence wide-angle X-ray diffraction (GIWAXD) for the $\mathrm{P}$ (NDI2OD-T2) polymer dried in between a top PDMS plate and Si substrate with separation of $1.5 \mu \mathrm{m}$ (Figure S5).

The GIWAXS result showed that the dried polymer adopted a face-on molecule packing on the substrate in comparison with a spin-coated polymer film (Supporting Information Note 8, Figure S9). This modified molecule configuration showed the significant influence of the hydrodynamic process during solution drying even under strong confinement. ${ }^{32,33}$

It is essential to balance the amplitudes of the two concentration-modulation waves for fabricating a well-defined 2D array. Several types of patterns are expected by tuning the wave amplitudes, wetting ability of substrates, and other experimental conditions. A summary of various potentially observable patterns is illustrated in Figure S10, including a 2D interference pattern, line patterns with different orientations, and dot array through dewetting of lines.

We have also carried out a similar experiment with $\mathrm{ZnO}$ nanoparticle $(100 \mathrm{~nm}$ in diameter) suspension in ethanol. In 
this case, only repeated pinning/depinning of contact line was found (Figure S11). The theory should also be applicalbe to colloids. In order to fabricate 2D array with colloids an optimized chain length of coating molecules is required to produce a proper spinodal region in the colloids/solvent phase diagram.

\section{Conclusions}

We demonstrated self-assembled 2D array patterns through the interference of two concentration-modulation waves in a drying solution next to a three-phase line. A 1DPS is formed by spinodal-precipitation along the contact line of a drying solution on a substrate through geometry confinement and hydrodynamic condensation. The generated 1DPS can be made to self-reproduce through the pinning-depinning of the contact line. The reproduced 1DPSs maintain the same phase with the initially formed 1DPS. The interference between the spinodalwave and the solute condensation wave at the contact line allows the formation of 2D patterns directly from the solutions with tuneable lattice parameters and lattice types. With further development, this work may open a new way to produce lowcost 2D self-assembled crystals for certain applications, such as opto-electric devices, ${ }^{34}$ lithography templates, ${ }^{35}$ and photonic materials. ${ }^{36,37}$

\section{Experimental}

The majority of samples used in this work were made via liquid confinement in wedge geometry as much stable liquid bridges are formed with such geometry. For sample preparation, solutions were introduced using a micropipette into the wedgeshaped spaces formed with silicon substrates ( $12 \mathrm{~mm} \times 12 \mathrm{~mm}$ ) and a glass cover plates $\left(12 \mathrm{~mm} \times 12 \mathrm{~mm}\right.$ ) with an angle of $25^{\circ}$. The joining of a substrate and a cover plate can be made with epoxy or scotch tape. The substrates were treated with $\mathrm{O}_{2}$ plasma (100 $\mathrm{W}$ for $5 \mathrm{~min}$ ) to increase the binding strength of the generated pattern with substrate. Liquid confinement in parallel plate geometry was done by introducing solution into space between substrates and parallel cover plates (glass or PDMS) that were separated by spacers made of plastic films with various thicknesses. For obtaining micro-size separation, PDMS stamps with patterned trench array were used as top coverage (Supplementary Fig.S5). For temperature dependence experiments, before introduction of solutions with micropipette the substrate/cover-plate wedges were placed on a digital hot-plate and thermally stabilised for three minutes. The AFM analysis was performed on Nanoscope III. GIWAXD measurements were conducted at the PLS-II 9A U-SAXS beamline of PAL in Korea. The $X$-rays from the in-vacuum undulator were monochromated (wavelength $=1.11 \AA$ ) using a double-crystal monochromator and focused both horizontally and vertically $[\mathrm{FWHM}=300 \mu \mathrm{m}(\mathrm{H}) \times 30 \mu \mathrm{m}(\mathrm{V})$ at the sample position] using K-B type mirrors. The GIWAXD system was equipped with a seven-axis motorized sample stage for the fine alignment of thin film. The sample-to-detector distance was 224 $\mathrm{mm}$, and diffraction patterns were recorded with a 2D chargecoupled device detector (Rayonix SX165).

\section{Conflicts of interest}

There are no conflicts to declare.

\section{Acknowledgements}

The authors would like to thank the UK Engineering and Physical Sciences Research Council (EPSRC) for the support through the EPSRC Centre for Innovative Manufacturing in Ultra Precision (EP/I033491/1).

\section{References}

1. C. D. Bain, E. B. Troughton, Y. T. Tao, J. Evall, G. M. Whitesides and R. G. Nuzzo, Journal of the American Chemical Society, 1989, 111, 321-335.

2. B. Dabbousi, C. Murray, M. Rubner and M. Bawendi, Chemistry of Materials, 1994, 6, 216-219.

3. Y. Yin and Y.-n. Xia, Advanced Materials, 2001, 13, 267-271.

4. M. Park, C. Harrison, P. M. Chaikin, R. A. Register and D. H. Adamson, Science, 1997, 276, 1401-1404.

5. S. O. Kim, H. H. Solak, M. P. Stoykovich, N. J. Ferrier, J. J. de Pablo and P. F. Nealey, Nature, 2003, 424, 411.

6. I. Bita, J. K. Yang, Y. S. Jung, C. A. Ross, E. L. Thomas and K. K. Berggren, Science, 2008, 321, 939-943.

7. T. Thurn-Albrecht, J. Schotter, G. Kästle, N. Emley, T. Shibauchi, L. Krusin-Elbaum, K. Guarini, C. Black, M. Tuominen and T. Russell, Science, 2000, 290, 2126-2129.

8. A. J. Hong, C.-C. Liu, Y. Wang, J. Kim, F. Xiu, S. Ji, J. Zou, P. F. Nealey and K. L. Wang, Nano letters, 2009, 10, 224-229.

9. O. Karthaus, N. Maruyama, X. Cieren, M. Shimomura, H. Hasegawa and T. Hashimoto, Langmuir, 2000, 16, 60716076.

10. H. Yabu and M. Shimomura, Chemistry of materials, 2005, 17, 5231-5234

11. H. Yabu, M. Takebayashi, M. Tanaka and M. Shimomura, Langmuir, 2005, 21, 3235-3237.

12. O. Karthaus, L. Gråsjö, N. Maruyama and M. Shimomura, Chaos: An Interdisciplinary Journal of Nonlinear Science, 1999, 9, 308-314.

13. H. Yabu and M. Shimomura, Advanced Functional Materials, 2005, 15, 575-581.

14. A. del Campo and E. Arzt, Chemical reviews, 2008, 108, 911945.

15. C. Lu and R. Lipson, Laser \& Photonics Reviews, 2010, 4, 568-580.

16. R. D. Deegan, O. Bakajin, T. F. Dupont, G. Huber, S. R. Nagel and T. A. Witten, Nature, 1997, 389, 827.

17. A. Cassie and S. Baxter, Transactions of the Faraday society, 1944, 40, 546-551.

18. Y. V. Kalinin, V. Berejnov and R. E. Thorne, Langmuir, 2009, 25, 5391-5397.

19. H. Bodiguel, F. Doumenc and B. Guerrier, The European Physical Journal Special Topics, 2009, 166, 29-32.

20. Z. Lin and S. Granick, Journal of the American Chemical Society, 2005, 127, 2816-2817.

21. J. Xu, J. Xia, S. W. Hong, Z. Lin, F. Qiu and Y. Yang, Physical review letters, 2006, 96, 066104. 
22. J. Xu, J. Xia and Z. Lin, Angewandte Chemie International Edition, 2007, 46, 1860-1863.

23. J. W. Cahn, The Journal of Chemical Physics, 1965, 42, 9399.

24. V. Bazhenov, Materials Science and Technology, 2015, 31, 1305-1312.

25. P. Van de Witte, P. J. Dijkstra, J. Van den Berg and J. Feijen, Journal of membrane science, 1996, 117, 1-31.

26. V. J. Klenin and S. L. Shmakov, Universal Journal of Materials Science, 2013, 1, 39-45.

$27 . \quad B$. Chen, E. E. Sigmund and W. Halperin, Physical review letters, 2006, 96, 145502.

28. A. Lipinski, W. Mycielski and G. Derfel, Molecular Crystals and Liquid Crystals, 1978, 41, 137-140.

29. E. Szwajczakaf and A. Szymański, molecular crystals and liquid crystals, 1986, 139, 253-261.

30. M. P. Longinotti and H. R. Corti, The Journal of Physical Chemistry B, 2009, 113, 5500-5507.

31. X. Wang and N. Mashita, Polymer, 2004, 45, 2711-2719.

32. Y. Diao, L. Shaw, Z. Bao and S. C. Mannsfeld, Energy \& Environmental Science, 2014, 7, 2145-2159.

33. J. A. Reinspach, Y. Diao, G. Giri, T. Sachse, K. England, Y. Zhou, C. Tassone, B. J. Worfolk, M. Presselt and M. F. Toney, ACS applied materials \& interfaces, 2016, 8, 17421751.

34. S. Li, J. Xu, W. Wang, I. Mathews, D. O'Mahony, Y. Xu and S. Roy, IEEE Transactions on Nanotechnology, 2014, 13, 537-540.

35. J. C. Hulteen and R. P. Van Duyne, Journal of Vacuum Science \& Technology A: Vacuum, Surfaces, and Films, 1995, 13, 1553-1558.

36. K. V. Sreekanth, K. H. Krishna, A. De Luca and G. Strangi, Scientific reports, 2014, 4, 6340.

37. N. Morimoto, S. Fujino, Y. Ito, A. Yamazaki, I. Sano, T. Hosoi, H. Watanabe and T. Shimura, Optics express, 2015, 23, 29399-29412. 